Kardiologe 2018 $12: 268-276$

https://doi.org/10.1007/s12181-018-0264-3

Online publiziert: 9 . Juli 2018

(c) Der/die Autor(en) 2018

CrossMark

\author{
M. Wiemer ${ }^{1}$ T. Schäufele ${ }^{2} \cdot$ T. Schmitz $^{3} \cdot$ S. Hoffmann ${ }^{4} \cdot$ T. Comberg $^{5}$. \\ H. Eggebrecht ${ }^{6} \cdot$ C. Langer ${ }^{7}$ \\ ' Klinik für Kardiologie und Internistische Intensivmedizin, Universitätsklinikum Johannes Wesling, Ruhr \\ Universität Bochum, Minden, Deutschland \\ ${ }^{2}$ Zentrum für Innere Medizin III - Kardiologie, Robert-Bosch-Krankenhaus, Stuttgart, Deutschland \\ ${ }^{3}$ Klinik für Kardiologie und Angiologie, Contilia Herz \& Gefässzentrum, Essen, Deutschland \\ ${ }^{4}$ Klinikum Barnim, Werner Forßmann Krankenhaus, Eberswalde, Deutschland \\ ${ }^{5}$ Klinik für Kardiologie und Angiologie II, Universitäres Herzzentrum Freiburg-Bad Krozingen, Freiburg-Bad \\ Krozingen, Deutschland \\ ${ }^{6}$ Cardioangiologisches Centrum Bethanien (CCB), Frankfurt am Main, Deutschland \\ ${ }^{7}$ Kardiologisch-Angiologische Praxis - Herzzentrum Bremen, Bremen, Deutschland
}

\title{
Herzkatheter: Diagnostik und Intervention über die Arteria radialis
}

\section{Standards und Technik}

Eine selektive Koronarangiographie erfolgte erstmals 1959 durch Mason Sones, der hierfür den Zugangsweg über die A. brachialis wählte. Trotz dieser frühen Pionierleistung verbreitete sich in den folgenden Jahren der durch Judkins und Amplatzer propagierte transfemorale Zugangsweg [28]. Ende der 1980erJahre trat der Zugangsweg über den Arm, nun über die Arteria (A.) radialis, in den Fokus - zunächst für die diagnostische Herzkatheteruntersuchung, dann zunehmend auch für Koronarinterventionen.

Die A. radialis verläuft im Bereich des Radiuskopfes sehr oberflächlich ohne weitere anatomische Strukturen in unmittelbarer Nähe, ist somit leicht aufzufinden und einfach zu komprimieren. $\mathrm{Ne}$ ben einem erheblichen Komfortgewinn des Patienten wurde auch den gefürchteten Blutungskomplikationen begegnet, wie sie insbesondere nach Interventionen unter Antikoagulationstherapie in der Leiste auftreten können $[19,25,53]$. Der transradiale Weg gilt auch bei hohem Blutungsrisiko als sicherster Zugang $[9,50]$. Für ältere Patienten, die häufig einen stark geschlängelten Gefäßverlauf

Stellungnahme im Auftrag der Arbeitsgemeinschaft Interventioneller Kardiologen (AGIK). bieten und vermehrt an einer peripheren arteriellen Verschlusserkrankung leiden, wurden in 2 randomisierten Studien bei transradialem Zugang signifikant weniger vaskuläre Komplikationen gezeigt als bei transfemoralem [1, 2, 24]. Die RIFLE-STEACS- und die MatrixStudie $[40,49]$ bestätigten diese Vorteile in einem großen Kollektiv von Patienten mit akutem ST-Hebungsinfarkt: Hier resultierte der transradiale Zugangsweg in einer geringeren Komplikationsrate und einer signifikanten Reduktion der Mortalität $[6,40]$. Der transradiale Zugang wurde daher in die STEMI-Leitlinien 2017 als Klasse-IA-Empfehlung aufgenommen $[18,39]$. Auch bei Patienten im kardiogenen Schock war ein transradiales Vorgehen von Vorteil [33].

Bei nachgewiesenen Vorteilen (Patientensicherheit, Komfort, Kostenreduktion) wird der transradiale Zugangsweg weiterhin kontrovers diskutiert. In Deutschland wird immer noch häufiger der transfemorale Zugang gewählt, aber der Anteil des Radialis-Zuganges steigt. Daten eines großen deutschen Registers von 2012 zeigen einen Radialis-Anteil von unter $20 \%$ [4]. Im Bericht über die Leistungszahlen der deutschen Herzka- theterlabore von 2015 findet sich eine Steigerung auf $37 \%$ [8].

Auch periphere Interventionen im Bereich der Becken/Beingefäße, insbesondere auch der Nierenarterien sind über den radialen Zugang möglich. Auf die Verwendung längerer Katheter muss dabei geachtet werden.

In dieser Arbeit sind Empfehlungen und Standards zur Patientenvorbereitung, Katheterauswahl, Durchführung der Untersuchung bzw. der Intervention zusammengestellt. Zudem wird auf mögliche Komplikationen und entsprechende Präventionen hingewiesen. Der dargestellte Expertenkonsens beruht neben der relevanten Literatur auch auf der klinischen Erfahrung der Autoren.

\section{Patientenauswahl und Vorbereitung}

Die transradiale Herzkatheteruntersuchung sollte in einer entspannten Atmosphäre erfolgen, was durch angepasste Helligkeit, ggf. Hintergrundmusik und eine leichte Sedierung erreicht werden kann. Transradial unerfahrene Untersucher sollten zu Beginn klinisch stabile Patienten mit gut palpablem Radialispuls auswählen. Für Anfänger ungünstig sind 


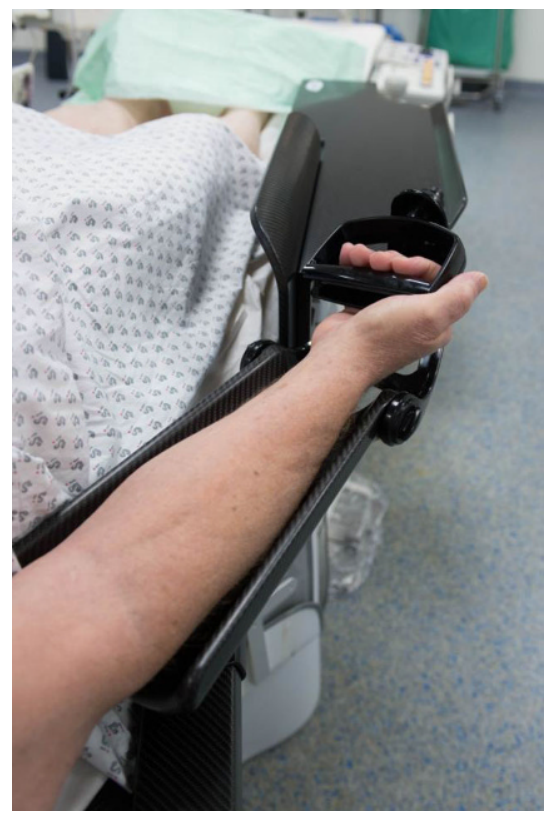

Abb. 1 \ Lagerung bei rechtsradialem Zugang mittels spezieller Extensionsschiene (Terumo Corporation, Tokio, Japan)

kleine, weibliche Patienten, ein bestehender Nikotinabusus sowie ein $\mathrm{Zu}$ stand nach koronarer Bypassoperation. Technische Schwierigkeiten sind auch bei älteren Patienten mit langjährigem Bluthochdruck zu erwarten, die häufig Gefäßschlängelungen in der Ellenbeuge und im Truncus (Tr.) brachiocephalicus aufweisen [14].

Auf eine transradiale Katheteruntersuchung sollte bei Patienten mit terminaler Niereninsuffizienz und (potenziellem) Shuntarm verzichtet werden. Auch das Raynaud-Syndrom stellt eine relative Kontraindikation dar [17].

Auf den früher empfohlenen AllenTests kann aufgrund aktueller Daten verzichtet werden [48]. Ein palpabler Radialispuls ist ausreichend [48]. Die ausgeprägte intraossäre Kollateralisierung des Hohlhandbogens führt dazu, dass mögliche Radialisverschlüsse meist asymptomatisch bleiben [16].

Durch die gute Komprimierbarkeit der A. radialis können auch intensiv antikoagulierte Patienten untersucht werden (INR-Wert bis 2,5 und nicht pausierte neue orale Antikoagulanzien). Aktuelle wissenschaftliche Daten gibt es dazu allerdings nicht, sodass patientenindividuell (Blutungsrisiko, erforderliche

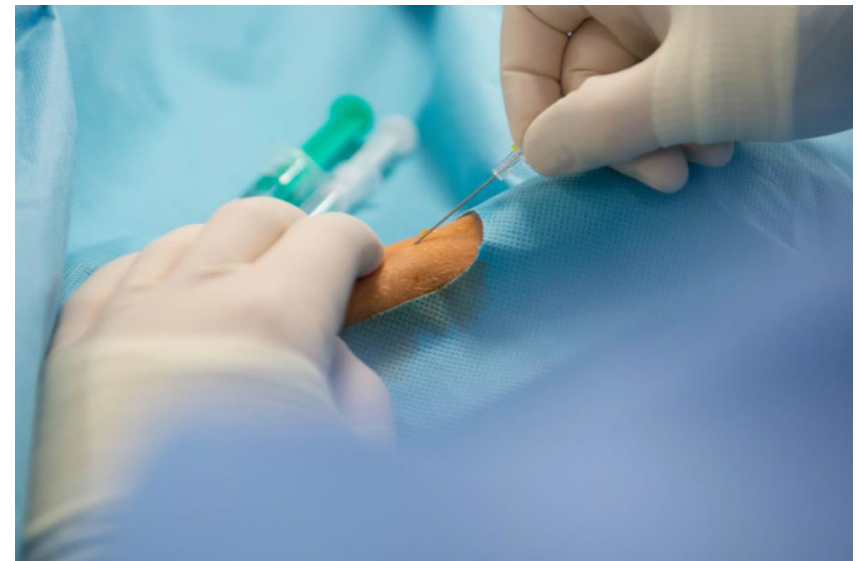

Abb. $2 \triangleleft$ Punktion der rechten $A$. radialis im flachen Winkel

PTCA, etc.) über die Durchführbarkeit des Eingriffs entschieden werden muss.

Der Arm des Patienten kann auf einem den Angiographietisch verbreiternden Brett möglichst nah am Körper gelagert werden. Erhältlich sind verschiedene Extensionsschienen (• Abb. 1).

Bei linksradialem Zugang wird der Arm nach erfolgter Punktion Richtung Körpermitte positioniert, sodass die weitere Prozedur von der rechten Herzkathetertischseite durchgeführt werden kann. Hierzu eignen sich Armschlingen oder große Kissen.

Für die Punktion wird der Arm des Patienten in einer überstreckten Supination in Dorsalfexion gelagert. Dafür kann die Hand z. B. mit einem Klettverschluss oder einem Pflaster passager fixiert werden. Zum Abdecken des Patienten stehen spezielle Abdecktücher zur Verfügung, die den transradialen und im Falle eines notwendigen Wechsels auch den transfemoralen Zugang ermöglichen.

\section{Punktion der Arteria radialis}

Der Punktionsort der Arterie liegt ungefähr $2 \mathrm{~cm}$ proximal des Processus styloideus. Nach der Sprühdesinfektion wird eine Lokalanästhesie mit 1-2 ml subkutan durchgeführt, wobei Irritationen des Gefäßes zu vermeiden sind. Alternativ ist die Anwendung von Lidocainpflastern möglich, die allerdings mindestens $1 \mathrm{~h}$ vor Untersuchung aufgebracht werden müssen. Die arterielle Punktion erfolgt in koaxialer Richtung in der Regel mit einer offenen, speziellen Stahlnadel (18-20 Gauge). Der Punktionswinkel ist
- im Gegensatz zur transfemoralen Punktion - eher flach (10-20 Grad) (• Abb. 2).

Radialispunktionssets und spezielle Plastikschleusen (ähnlich einer Venüle), die alternativ verwendet werden können, sind kommerziell erhältlich.

Bei ausreichendem (pulsierendem) arteriellen Rückfluss wird ein gerader Draht in das Gefäß eingeführt. Dabei ist darauf zu achten, dass der Draht ohne jeden Widerstand vorgebracht werden kann.

Danach wird eine Radialisschleuse in die A. radialis eingebracht. Die Schleusen zeichnen sich durch eine langgezogene stark verjüngende Spitze sowie eine spezielle hydrophile Beschichtung aus, die die irritierende Reibung des Gefäßes reduziert. Aufgrund ihres geringen $\mathrm{Au}$ ßendiameters verringert die Glidesheath Slender ${ }^{\odot}$ Schleuse (Terumo Corporation, Tokio, Japan) die Gefäßirritation [3]. Ob längere Schleusen (z.B. $23 \mathrm{~cm}$ ) Vorteile haben, ist bisher nicht hinreichend belegt.

Sind großlumige Führungskatheter notwendig, ist auch ein schleusenloses Intervenieren möglich (z.B. Railway ${ }^{\circledR}$ Sheathless Access System, Cordis Corporation, Miami, USA).

\section{Spasmusprävention}

Die Punktion und das Einführen der Schleuse können zu Vasospasmen der A. radialis führen. Zur medikamentösen Prophylaxe kann neben einer Sedierung (s. oben) eine individualisierte Dosierung von Nitraten (z.B. 0,2 mg) und/ oder einem Kalziumantagonisten (z.B. Verapamil 2,5 mg) ggf. auch in Kombi- 
nation über die liegende Schleuse verabreicht werden. Der Blutdruck und entsprechende Kontraindikationen sind $\mathrm{zu}$ beachten [41].

\section{Periprozedurales Gerinnungs- management}

Zur Vermeidung eines A. radialis-Verschlusses (s. Abschnitt „Komplikationen“) ist eine adäquate Antikoagulation essenziell. Diese kann zu Beginn der Untersuchung durch die Gabe unfraktionierten Heparins (Standarddosis für eine diagnostische Prozedur 5000I.E.) oder mit niedermolekularem Heparin (z.B. Enoxaparin $60 \mathrm{mg}$ ) erreicht werden [37, 42]. Diskutiert wird, dass eine intraarterielle Heparingabe aufgrund der Säureeigenschaften zu einer Reizung der A. radialis führen kann, was klinisch allerdings nicht bedeutsam erscheint [30]. Bei einer folgenden Intervention ist die Heparindosis anzugleichen, ggf. nach Messung der ACT („activated clotting time“, Zielkorridor 250-350s). Im Fall einer Heparin-induzierten Thrombozytämie erfolgt die Gabe von Faktor Xa oder Thrombinantagonisten. Eine vorbestehende Antikoagulation ist bei der Heparindosierung bzw. alternativen Substanzen zu berücksichtigen.

\section{Zugang und Katheterauswahl}

\section{Zugang}

Für Nativgefäße ist der rechts-radiale $\mathrm{Zu}$ gangsweg Standard. Für die A. radialis rechts spricht die ergonomisch günstigere Arbeitsposition am Kathetertisch. Im Fall eines Versagens ist ein Wechsel auf die linke A. radialis möglich (s. Abschnitt „Vorbereitung“).

Für den linksradialen Zugangsweg spricht, dass das Katheterverhalten in der Aorta ascendens dem transfemoralen Vorgehen ähnelt (• Abb. 3).

Bei der Sondierung von Bypassgefäßen ist die Darstellung venöser Grafts über den rechtsradialen Zugang ohne größere Probleme möglich [23]. Bei der selektiven Sondierung der A. mammaria interna links (LIMA) sollte der linksradiale Zugang bevorzugt werden.

Kardiologe 2018 $\cdot 12: 268-276$ https://doi.org/10.1007/s12181-018-0264-3

(c) Der/die Autor(en) 2018

\section{Wiemer · T. Schäufele · T. Schmitz · S. Hoffmann · T. Comberg · H. Eggebrecht · C. Langer Herzkatheter: Diagnostik und Intervention über die Arteria radialis. Standards und Technik}

\section{Zusammenfassung}

Die Koronardiagnostik oder Intervention über die A. radialis führt zu einer schnelleren Mobilisation des Patienten und somit auch zu kürzeren Krankenhausaufenthalten im Vergleich zum femoralen Zugang. Durch die oberflächliche Lage der Arterie ist die Punktion und Kompression einfach und reduziert vaskuläre Blutungskomplikationen signifikant. Dies hat bei einem ST-Hebungsinfarkt mit intensivierter Antikoagulation zu einer Mortalitätsreduktion und dadurch in den aktuellen Leitlinien zu einer KlasseIA-Empfehlung geführt. Vorbereitung, Punktion, Katheterwahl und der Ablauf des
Herzkatheters unterscheiden sich nur geringfügig vom femoralen Zugangsverfahren. Nach einer Lernkurve und bei häufigerer Anwendung sind mögliche Nachteile wie Spasmen, Strahlenbelastung oder radiale Gefäßverschlüsse vernachlässigbar. In dieser Arbeit sind Empfehlungen und Standards zur Durchführung der Untersuchung bzw. der Intervention über die A. radialis zusammengestellt.

Schlüsselwörter

Herzkatheter · Koronarintervention · Radialer Zugang $\cdot$ Technik $\cdot$ Prognose

\section{Cardiac catheter: diagnostics and intervention via radial access. Standards and technique}

\section{Abstract}

Using the radial approach for coronary angiography and interventions results in faster mobilization and early discharge of patients compared to femoral access. Due to the superficial position of the radial artery, puncture and compression is easy and significantly reduces bleeding complications. In patients with ST-elevation myocardial infarction and intensive anticoagulation therapy, radial access improves outcome, leading to a class IA recommendation of the recent European Society of Cardiology (ESC) guidelines. Preprocedural preparation, puncture, cannulation, catheter choice and handling differ only slightly from the femoral approach. After a short learning curve and with regular implementation of radial access, possible drawbacks, such as vasospasm, radiation exposure and radial occlusion are negligible. This article provides recommendations, standards and techniques for a safe cardiovascular intervention via radial access.

\section{Keywords}

Catheterization - Cardiovascular intervention . Radial approach · Technique · Prognosis
In Fällen, in denen eine Sondierung der Brustwandarterien von ipsilateral nicht möglich ist, wird der transfemorale Zugangsweg empfohlen. Bei der erforderlichen selektiven Darstellung beider Arteriae mammariae ist der transfemorale Zugang die Methode der Wahl.

Zur Einführung der Katheter empfiehlt sich die Verwendung eines Standard 0,035-inch-Drahtes $(0,889 \mathrm{~mm})$ mit J-Tip (J-Draht). Für sehr kleine Gefäße wird ein Mini-J-Tip-Draht angeboten. Bei sehr gewundenen Gefäßverläufen („Kinking“) und Gefäßschlingen („Loops“) ist der Wechsel auf einen hydrophil beschichteten Draht (z.B. Glidewire $^{\oplus}$, Terumo Corporation, Tokio, Japan) hilfreich, der unter Durchleuchtung vorgeschoben werden sollte. Ist das Vorbringen des Katheters in der A. radialis oder brachialis problematisch, sollte eine angiographische Darstellung der Gefäßstruktur erfolgen.

In der Mehrheit der Fälle gelingt eine Drahtpassage in die Aorta direkt oder mithilfe tiefer Inspiration. Bei Abweichen in die Aorta descendens kann durch die Steuerung des nachgeschobenen Katheters (Rotation, Rückzug) die Aorta ascendens sondiert werden. 


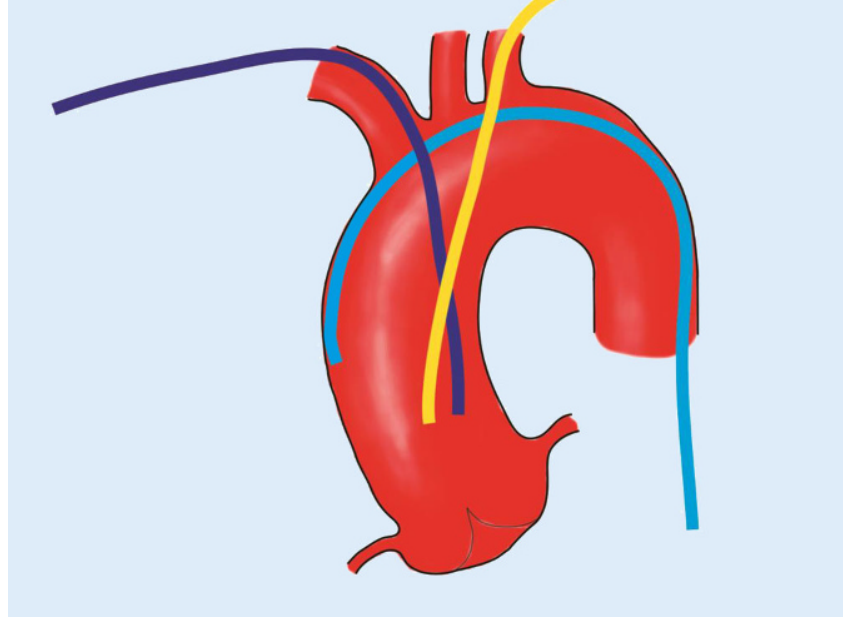

Abb. $3<$ Typische Katheterlage im Bereich des Aortenbogens und der Aorta ascendens bei rechts-(dunkelblau) und links-radialem (gelb) bzw. transfemoralem Zugang (hellblau)

\section{Alternativer Zugang}

Der Zugang über die A. ulnaris wird nur sehr selten genutzt. Da das Gefäß sehr früh tief in die Muskelloge zieht, ist die Kompression schwieriger als bei der oberflächlich verlaufenden Radialarterie.

Eine weitere erst kürzlich vorgestellte Zugangsoption ist die distale A. radialis. Der Punktionsort ist ein palpabler Puls in der Foveola radialis (Tabatière oder auch Snuff-Box genannt). Die Erfahrungen hier sind noch limitiert. Ein möglicher Vorteil kann eine geringere Rate an Radialis-Verschlüssen sein. Die postprozedurale Kompression muss bisher durch einen individuell angelegten Verband erfolgen.

\section{Katheterauswahl Diagnostik}

Prinzipiell ist für den transradialen $\mathrm{Zu}$ gang kein spezielles Kathetermaterial notwendig, d.h. es können Standarddiagnostikkatheter von 4-6 French (Fr.) benutzt werden [7].

Das rechts-koronare Ostium ist meist mit einem Judkins rechts (JR) 4,0 Katheter zu sondieren. Bei besonderen anatomischen Bedingungen können auch der Amplatz-links-I(AL I)-, Multi-Purpose(MP)- oder der Amplatz-rechts(AR)Katheter benutzt werden [7]. Die Intubation des links-koronaren Ostiums gelingt bei normalen anatomischen Verhältnissen mit einem Standard-Judkinslinks(JL)-3,5-Katheter.
Generell ist bei transradialem Zugang und Intubation der linken Kranzarterie mit Judkins-Kathetern eine kleinere Größe als bei transfemoralem Vorgehen zu wählen. Auch hier können bei atypischer Anatomie Alternativkatheter (z.B. AL I, AL II) ähnlich wie von femoral eingesetzt werden.

Zur Vermeidung von mechanischen Irritationen (Katheterwechsel), die zu Vasospasmen führen können, bzw. mit Hinblick auf eine mögliche Zeit- und Kostenersparnis ist ein Ein-KatheterKonzept von Vorteil (z.B. TIGER II ${ }^{\odot}$, Terumo Corporation, Tokio, Japan). Mit derartigen Kathetern gelingt sowohl die Sondierung des rechts- als auch des linkskoronaren Ostiums [22]. Dabei wählt man für Patienten mit einer Körpergröße kleiner als $175 \mathrm{~cm}$ in der Regel die Größe 3,5, größer $175 \mathrm{~cm}$ eine 4,0 Größe [20].

Liegt ein Zustand nach transkatheterimplantierter Aortenklappe (TAVI) vor, insbesondere bei Koronarostien-überdeckenden oder hochpositionierten Prothesen, ist der Zugang zu den Koronarostien oftmals durch die Stentstreben erschwert. Kleinere Amplatz-Katheter aber auch die Judkins-Katheter (insbesondere für die linke Herzkranzarterie) sind eine mögliche Option.

Bypässe: Zur Darstellung der Venenbypässe von rechts-radial werden die gleichen Katheter wie von femoral verwendet. Ein hochabgehender Venenbypass ist mit einem AL 1 oder AL 2 gut zu sondie- ren (•Abb. 4). Links-radial sind die Venenbypässe mit den Standardkathetern leichter zu erreichen. Zur Sondierung des LIMA-Bypasses kann bei links-radialem Zugang der Mammaria-Katheter benutzt werden.

\section{Katheterauswahl Intervention}

Der überwiegende Anteil der Koronarinterventionen kann mittels 5-Fr.- bzw. 6-Fr.-Führungskathetern sicher und erfolgreich durchgeführt werden [51]. Für wenig komplexe Interventionen ist bei transradialem Zugang die Verwendung von 5-Fr.-Führungskathetern zu bevorzugen, da hierdurch Verletzungen und Irritationen der kleinkalibrigen A. radialis minimiert werden. Durch tiefe Intubation der Koronararterie („deep seating“) kann der Backup-Support verbessert werden. Bei komplexeren Koronarinterventionen (z.B. Bifurkationsstenosen mit Kissing-balloon-Notwendigkeit) oder Einsatz spezieller Verfahren (z.B. Scaffold-Implantation, Rotablation) ist ein 6-Fr.- oder 7-Fr.-Führungskatheter erforderlich. Durch die Verwendung der oben genannten Glidesheath Slender ${ }^{\circledR}$ Schleusen (Terumo Corporation, Tokio, Japan) ist auch bei diesen Größen ein transradiales Vorgehen in der Regel problemlos.

Bei reduziertem Back-up sollten Führungskatheter mit einer extra Back-upKonfiguration (wie z.B. EBU oder XB) verwendet werden. Bei der Wahl der Größe der XB- bzw. EBU-Kurve wird eine kleinere Größe als beim transfemoralen Vorgehen bevorzugt. Der EBU-3,0-Katheter wird bei sehr kleinem Anulusdurchmesser empfohlen.

Bei rechts-koronaren Interventionen ist bei unkomplizierter Anatomie ein JRFührungskatheter in der Regel ausreichend. Sollte mehr Back-up notwendig sein, empfiehlt sich eine AL-Konfiguration (üblicherweise AL1 oder AL 0,75), da der AL-Katheter sich zusätzlich an der kontralateralen Aortenwand abstützt. Bei Intervention im Bereich des rechts-koronaren Ostiums ist der JR-Katheter eine gute Wahl, der leichter semiselektiv an das Ostium herangeführt werden kann und so eine exakte Stentplatzierung bis 


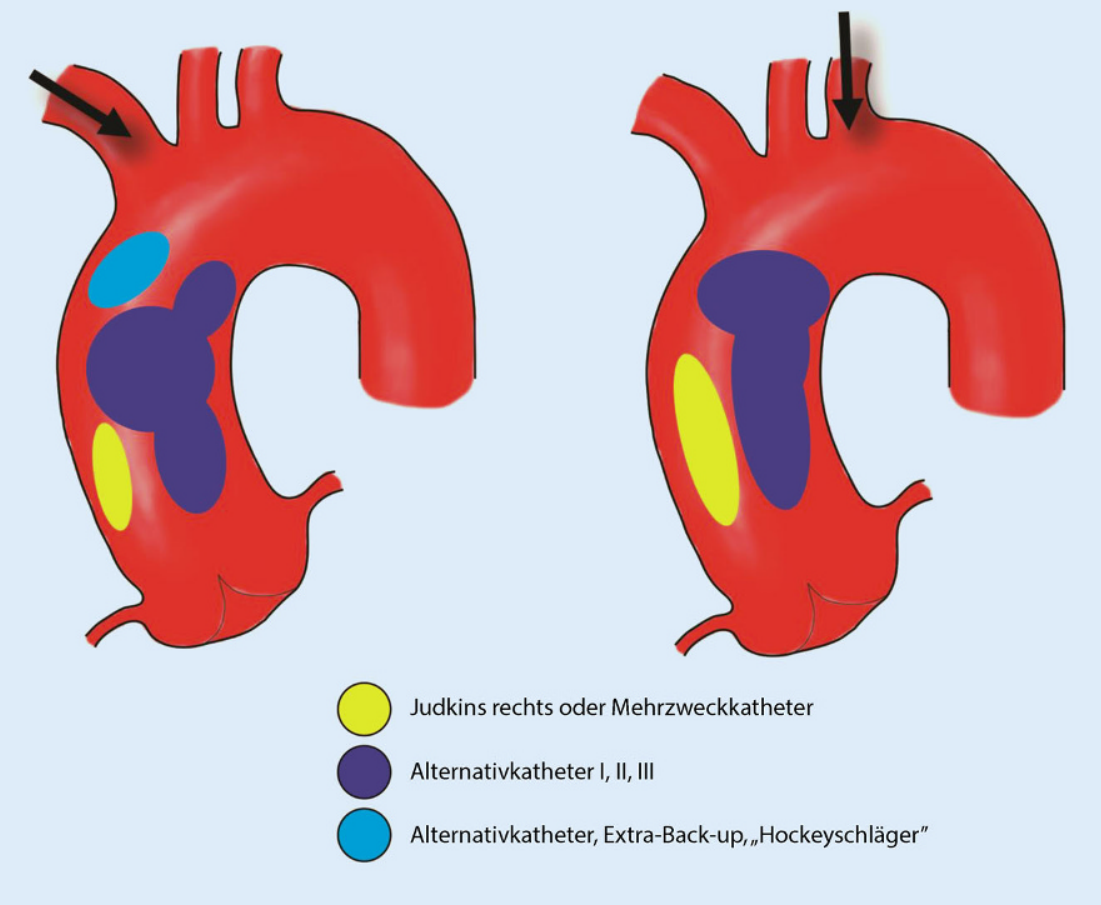

Abb. 4 \ Katheterauswahl rechts- und links-radial in Abhängigkeit von der Lokalisation der Venenbypässe

an den aortalen rechtskoronaren Abgang möglich ist.

Für Interventionen von aortokoronaren Bypässen zur linken Koronararterie sind AL-Konfigurationen, zur rechten Koronararterie Multipurpose-Katheter vorteilhaft. Interventionen eines Insitu-RIMA- oder LIMA-Bypasses sollten jeweils über einen ipsilateralen $\mathrm{Zu}$ gang mittels Mammaria-Katheter erfolgen. Hierbei kann eine Katheterlänge von $90 \mathrm{~cm}$ ausreichend sein.

Bei unzureichendem Back-up eines Führungskatheters wird die Katheterlage durch Nutzung von 2 Drähten („buddy wire"), die Verwendung eines Ankerballons oder die Anwendung der MotherChild-Technik stabilisiert.

\section{Strahlenexposition}

Gegenüber dem transfemoralen Zugang ist bei transradialem Zugang mit einer geringen, aber signifikant erhöhten Strahlenbelastung zu rechnen, wenngleich die Interpretation der Daten kontrovers diskutiert wird [12, 21, 32]. Bei Verwendung des linksradialen Zugangs ist die geringere Strahlenexposition mit der günstigeren Anatomie der A. subclavia links im

Um eine Intervention über die A. radialis
Untersuchungs- und Durchleuchtungsdauer [45].

\section{Komplexe Zugangswege}

Das vergleichsweise kleine Gefäßlumen der A. radialis ist bei der Sondierung mit Drähten oder Kathetern verletzungsanfälliger. Es gibt eine Vielzahl anatomischer Verlaufsvarianten insbesondere im Übergangsbereich der A. radialis in die A. brachialis, die die Sondierung der Aorta ascendens erschweren können. Grundsätzlich gilt, dass beim Versuch der Gefäßsondierung mit Drähten bei einem Widerstand sehr vorsichtig vorgegangen werden muss. Das Manöver an der nicht überwindbaren Stelle kann Schmerzen verursachen. Die Drahtpassage hat an dieser Stelle unter Durchleuchtung zu erfolgen. Ein hydrophiler Draht (Glidewire ${ }^{\Theta}$; Terumo Corporation, Tokio, Japan), aber auch dünnere Sondierungsdrähte (z.B. „floppy“ PTCA-Drähte) können dabei hilfreich sein. Nach Überwinden des Hindernisses ist das weitere Vorschieben meist unproblematisch.

Schwierig kann die Passage von Loops sein. Ein Vorbringen von PTCA-Drähten gelingt meist einfacher, das Nachführen des Katheters hingegen kann problematisch sein. Als Hilfsmittel kann ein PTCA-Ballon, der aus der Spitze des Katheters hinausragt, unter Durchleuchtung über den Loop vorgeschoben werden („,ballon-assisted-tracking“; - Abb. 5). Bei erfolglosen Manövern muss ggf. der Zugangsweg gewechselt werden.

Die Sondierung der Aorta ascendens kann durch Kinking im Truncus brachiocephalicus, bei elongiertem Aortenbogen oder einer Gefäßanomalie (z. B. A. lusoria) erschwert sein. Unter tiefer Inspiration hilft ein hydrophil beschichteter oder auch ein steiferer Draht bei der Passage. Bei einem notwendigen Katheterwechsel sollten ausreichend lange Drähte zur Verfügung stehen, um den erreichten $\mathrm{Zu}$ gangsweg zu sichern. komplikationsfrei durchzuführen, ist ein regelmäßiger Einsatz des Zugangsweges notwendig. Mindestens 80 Prozeduren pro Untersucher und Jahr korrelieren mit einer Reduktion der Komplikationen, der

\section{Spasmus}

Die A. radialis neigt im Vergleich zur A. femoralis vermehrt zu Vasospasmen, die zum Abbruch der Untersuchung 


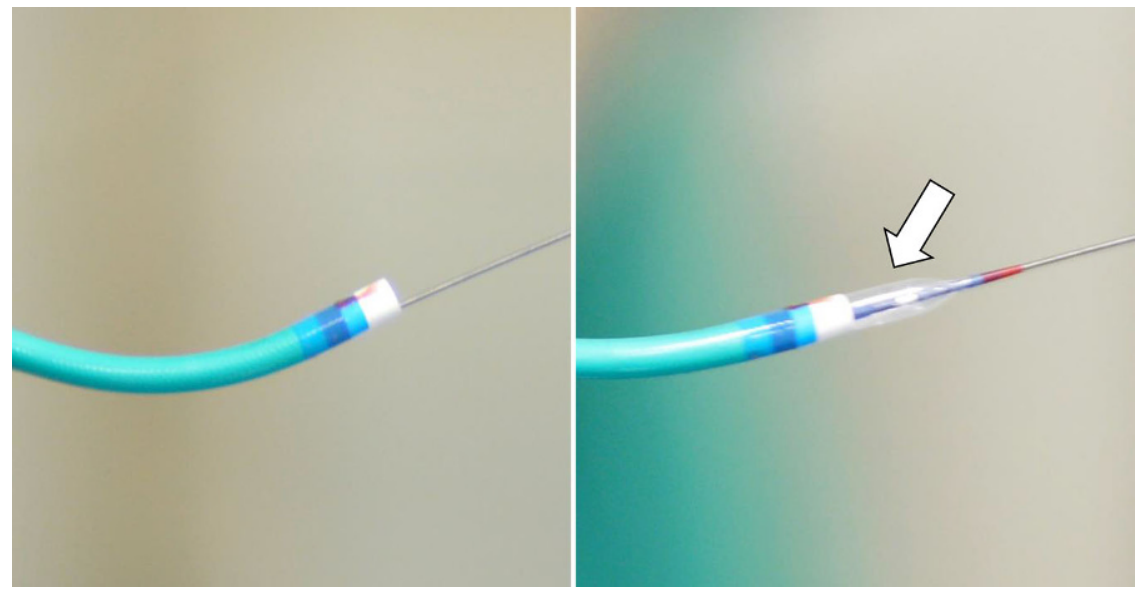

Abb. 5 A „Ballon-assisted-tracking“: Bei Passagebehinderung wird ein aus der Katheterspitze herausragender Ballon (s. Pfeil) inflatiert, sodass er eine (weniger traumatische) Läsions- oder Gefäßpassage ermöglicht

führen können. Mit zunehmender Routine und Erfahrung in der transradialen Untersuchungstechnik treten Spasmen deutlich seltener auf. Eine vermehrte Neigung besteht bei jüngeren, weiblichen Patienten. Ein Nikotinkonsum kann das Auftreten von Spasmen verstärken. Vorbeugend ist eine Nikotinkarenz, eine beruhigende Patientenführung während der Vorbereitung, sowie während des Eingriffs. Angespannte Patienten sollten durch orale oder intravenöse Gabe eines Sedativums behandelt werden (s. Abschnitt „Patientenvorbereitung“). Ist das Vorbringen eines großlumigeren Katheters aufgrund eines Spasmus erschwert, so helfen die Gabe von Nitraten und/ oder Kalziumantagonisten (s. Abschnitt "Spasmusprävention") sowie eine intensivierte Analgesie und das „balloonassisted-tracking“ (s. Abschnitt „Komplexe Zugangswege").

\section{Gefäßperforation}

Beim Einsatz des Sondierungsdrahtes können neben dem relativ kleineren Gefäßkaliber der Radialarterie auch kleinere Seitenäste, komplexe Verzweigungen und Gefäßloops vor Einmündung in die A. brachialis problematisch sein (s. Abschnitt "Komplexe Zugangswege"). Die Drahtsondierung nach erfolgreicher Punktion muss daher vorsichtig und ohne jeglichen Widerstand erfolgen.

Im Falle einer Gefäßperforation sollte der Versuch unternommen werden, mit-

\section{Zerebrale Komplikationen}

Ob der transradiale Zugang zu vermehrten zerebralen Ereignissen führt, wird in der Literatur kontrovers diskutiert [26]. In einer Metaanalyse [19] fand sich bei transradial untersuchten Patienten kein erhöhtes Risiko für zerebrale Ereignisse, was durch die großen Studien MATRIX und RIVAL bestätigt wurde [19, 49] und auch bei über 75-jährigen Patienten gezeigt werden konnte [1].

\section{Radialisverschluss}

Die in älteren Studien angegebenen Verschlussraten der A. radialis bewegen sich zwischen 5 und $11 \%$ [29,52]. In aktuellen Untersuchungen, die effektive präventive Maßnahmen berücksichtigen, liegt die Verschlussrate unter 1\% [31]. Der Verschluss der A. radialis verläuft in der Regel asymptomatisch. Beschwerden können als Hypästhesie, schnellere muskuläre Ermüdbarkeit und Schmerzen im Bereich der betroffenen Hand auftreten. Mit einer spontanen Rekanalisation kann in über $50 \%$ der Verschlussfälle gerechnet werden [46]. Zu den präventiven Maßnahmen zur Verhinderung eines Gefäßverschlusses gehören die oben beschriebene obligate Gabe von mindestens $5000 \mathrm{E}$ Heparin und das Arbeiten mit möglichst kleinen Schleusen und Kathetern [47]. Eine diagnostische Angiographie gelingt nahezu immer unter Verwendung von 4-F- und 5-F-Kathetern. Bei vorhersehbar langwierigen Interventionen mit sehr langer Liegezeit von Schleuse und Katheter (z.B. Rekanalisation eines chronischen Koronarverschlusses) muss die Verwendung des transradialen Zugangs kritisch abgewogen werden.

Unverzüglich nach dem Eingriff sollte die Schleuse entfernt werden (s. Abschnitt „Nachsorge“). Beim Anlegen des Druckverbandes ist das Prinzip der „patent hemostasis" zu beachten, also des erhaltenen antegraden Flusses, bei erreichter Hämostase. Im Falle eines Radialisverschlusses ist durch medikamentöse Intervention unter Einsatz niedermolekularen Heparins eine Wiedereröffnung möglich [47]. Inwieweit Vitamin-K-Antagonisten oder neue orale Antikoagulanzien über einen bestimmten Zeitraum (z. B. 4 Wo- 


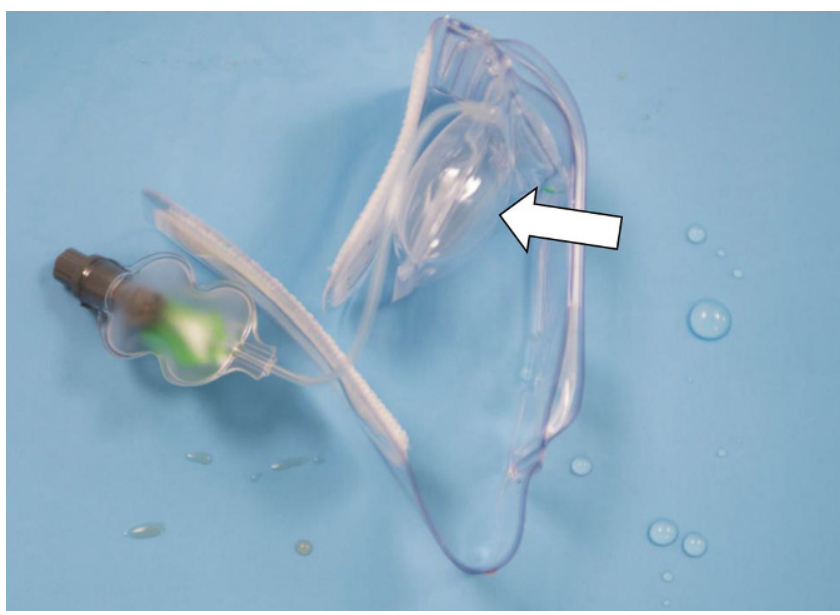

Abb. $6 \Delta$ Kompression der Punktionsstelle mit transparenter Manschette mit regulierbarem Luftkissen (TR Band ${ }^{\circledR}$, Terumo Corporation, Tokio, Japan), s. Pfeil

chen oder auch länger) Erfolg versprechend sind, ist durch wissenschaftliche Daten nicht hinreichend belegt.

Eine zeitlich begrenzte Kompression der A. ulnaris erzeugt eine Hyperperfusion im Radialisstromgebiet mit der Chance einer Reperfusion [5].

\section{Nachsorge}

Nach Beendigung der transradialen Herzkatheteruntersuchung wird der Katheter mit Draht zurückgezogen. Anschließend sollte die arterielle Schleuse noch auf dem Kathetertisch entfernt werden. Ein Belassen der Schleuse ist unbedingt $\mathrm{zu}$ vermeiden, da dies eine erhöhte Radialisverschlussrate zur Folge hat (s. Abschnitt „Komplikationen“) [10]. Es gibt unterschiedliche Möglichkeiten der anschließenden mechanischen Kompression der Punktionsstelle. Entscheidend ist das Prinzip der „patent hemostasis“ (s. Abschnitt „Radialisverschluss“). Auf dem Markt verfügbar sind unterschiedliche Verschlusssysteme. Es gibt nur wenige wissenschaftliche Arbeiten über den Vergleich der Systeme untereinander [11,38], sodass eine adäquate Wertung hinsichtlich der Qualität nicht möglich ist.

In Deutschland weit verbreitet ist der Einsatz des TR-Bandes ${ }^{\circledR}$, (Terumo Corporation, Tokio, Japan), eine aus transparentem Plastik bestehende Manschette mit Klettverschluss und einem dem Druckaufbau dienenden regulier-

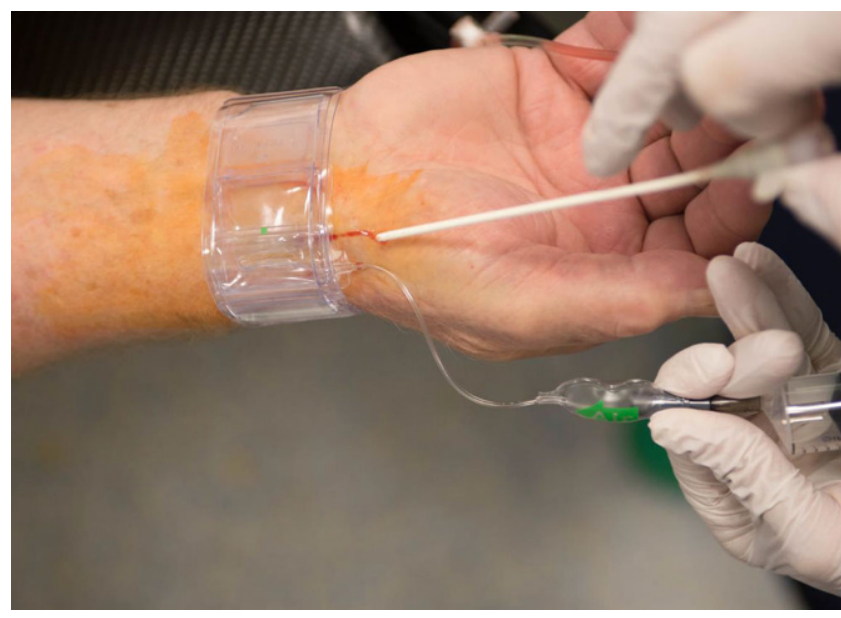

Abb. 7 A Anlage des TR Bandes ${ }^{\circledR}$ und Entfernung der Schleuse

baren Luftkissen (• Abb. 6). Im Vergleich zur konventionellen Kompression haben sich damit weniger Radialisverschlüsse gezeigt [35].

Nach Anlage des TR-Bandes und Entfernung der Schleuse (• Abb. 7) wird die in das Polster injizierte Luft so lange abgelassen, bis etwas Blut aus der Punktionsstelle austritt. Dann werden ca. $2 \mathrm{ml}$ Luft wieder hinzugegeben, um so einen für den Patienten optimalen „individualisierten“ Verschlussdruck zu erreichen. Idealerweise wird die Perfusion pulsoxymetrisch kontrolliert („patent hemostasis“). [37]. Der Kompressionsdruck sollte im Verlauf von 2-4 h sukzessive reduziert werden. Durch eine Reduktion der Kompressionsdauer kann die Rate an Radialisverschlüssen gesenkt werden [34].

Klagt der Patient über Schmerzen, sollte sichergestellt werden, dass keine Blutung und keine Ischämie aufgetreten sind (s. Abschnitt Komplikationen). Gegebenenfalls kann zur Druck und Schmerzentlastung etwas Luft abgelassen werden. Eine Objektivierung der Fingerdurchblutung ist durch ein Pulsoxymeter(s.oben) möglich. BeiNachblutung, z. B. aufgrund einer intensivierten Antikoagulation, muss das Kompressionsintervall verlängert werden.

Neben Nachblutungen, unter Umständen mit Ausbildung eines Hämatoms, können durch die Bandage Schmerzen im Punktionsbereich oder im Hand/Fingerbereich sowie Gefühlstörungen (Taubheitsgefühl, Kribbelparäs- thesien) auftreten. Eine livide Verfärbung der Akren, bedingt durch den behinderten venösen Rückstrom, ist klinisch meist unbedeutend.

Vor Entlassung sollte die Hand inspiziert und der Radialispuls palpatorisch geprüft werden. Bei Verdacht auf Mangeldurchblutung sind eine Pulsoxymetrie und/oder eine Doppler-/ Duplexuntersuchung anzuschließen.

In seltenen Fällen kann es zu lokalen Entzündungen im Bereich der Einstichstelle, zu Druckstellen bis hin zu Nekrosen und der Ausbildung von Aneurysmata kommen. In wenigen Fällen ist ein Complex-Regional-Pain-Syndrom beschrieben [35].

Durch den beschriebenen Druckverband im Handgelenkbereich sind eine schnellere Mobilisation mit hoher $\mathrm{Pa}$ tientenzufriedenheit [25] sowie kürzere Krankenhausaufenthalte im Vergleich zum femoralen Zugang hinreichend belegt $[13,27]$.

\section{Korrespondenzadresse}

PD Dr. med. M. Wiemer
Klinik für Kardiologie und
Internistische Intensivme-
dizin, Universitätsklinikum
Johannes Wesling, Ruhr
Universität Bochum
Hans-Nolte-Str. 1,
32429 Minden, Deutschland
marcus.wiemer@
muehlenkreiskliniken.de




\section{Einhaltung ethischer Richtlinien}

Interessenkonflikt. M. Wiemer, T. Schäufele, S. Hoffmann, T. Comberg, H. Eggebrecht und C. Langer geben an, dass kein Interessenkonflikt besteht. T. Schmitz weist auf folgende Beziehung hin: Vortragshonorare Terumo.

Dieser Beitrag beinhaltet keine von den Autoren durchgeführten Studien an Menschen oder Tieren.

Open Access Dieser Artikel wird unter der Creative Commons Namensnennung 4.0 International Lizenz (http://creativecommons.org/licenses/by/4.0/deed. de) veröffentlicht, welche die Nutzung, Vervielfältigung, Bearbeitung, Verbreitung und Wiedergabe in jeglichem Medium und Format erlaubt, sofern Sie den/die ursprünglichen Autor(en) und die Quelle ordnungsgemäß nennen, einen Link zur Creative Commons Lizenz beifügen und angeben, ob Änderungen vorgenommen wurden.

\section{Literatur}

1. Achenbach $S$, Ropers $D$, Kallert L, Turan $N$, Krahner R, Wolf T, Garlichs C, Flachskampf F, Daniel WG, Ludwig J (2008) Transradial versus transfemoral approach for coronary angiography and intervention in patients above 75 years of age. Catheter Cardiovasc Interv 72:629-635. https:// doi.org/10.1002/ccd.21696

2. Alnasser SM, Bagai A, Jolly SS, Cantor WJ, Dehghani P, Rao SV, Cheema AN (2017) Transradial approach for coronary angiography and intervention in the elderly: A meta-analysis of 777,841 patients. Int J Cardiol 228:45-51. https://doi.org/10.1016/j. ijcard.2016.11.207

3. Aminian A, Dolatabadi D, Lefebvre $P$, Zimmerman R, Brunner P, Michalakis G, Lalmand J (2014) Initial experience with the Glidesheath Slender for transradial coronary angiography and intervention: a feasibility study with prospective radial ultrasound follow-up. Catheter Cardiovasc Interv 84:436-442. https://doi.org/10.1002/ccd.25232

4. Bauer T, Hochadel M, Brachmann J, Schachinger V, Boekstegers P, Zrenner B, Zahn R, Zeymer U (2015) Use and outcome of radial versus femoral approach for primary $\mathrm{PCl}$ in patients with acute ST elevation myocardial infarction without cardiogenic shock: results from the ALKK $\mathrm{PCl}$ registry. Catheter Cardiovasc Interv 86(Suppl 1):S8-S14. https://doi. org/10.1002/ccd.25987

5. Bernat I, Bertrand OF, Rokyta R, Kacer M, Pesek J, Koza J, Smid M, Bruhova H, Sterbakova G, Stepankova L, Costerousse O (2011) Efficacy and safety of transient ulnar artery compression to recanalize acute radial artery occlusion after transradial catheterization. Am J Cardiol 107:1698-1701. https://doi.org/10.1016/j.amjcard.2011.01.056

6. Bernat I,HorakD, Stasek J, Mates M, PesekJ, Ostadal P, Hrabos V, Dusek J, Koza J, Sembera Z, Brtko M, Aschermann O, Smid M, Polansky P, Al Mawiri A, Vojacek J, Bis J, Costerousse O, Bertrand OF, Rokyta R (2014) ST-segment elevation myocardial infarction treated by radial or femoral approach in a multicenter randomized clinical trial: the STEMIRADIAL trial. J Am Coll Cardiol 63:964-972. https:// doi.org/10.1016/j.jacc.2013.08.1651

7. Bertrand OF, Rao SV, Pancholy S, Jolly SS, RodesCabau J, Larose E, Costerousse O, Hamon M, Mann T (2010) Transradial approach for coronary angiography and interventions: results of the first international transradial practice survey. Jacc Cardiovasc Interv 3:1022-1031. https://doi.org/ 10.1016/j.jcin.2010.07.013

8. Bestehorn K, Bauer T, Fleck E, Bestehorn M, Pauletzki J, Hamm C (2015) Coronary procedures in German hospitals: a detailed analysis for specific patient clusters. Clin Res Cardiol 104:555-565. https://doi.org/10.1007/s00392-015-0818-3

9. Biancari F, D'Andrea V, Di Marco C, Savino G, Tiozzo V, Catania A (2010) Meta-analysis of randomized trials on the efficacy of vascular closure devices after diagnostic angiography and angioplasty. Am Heart J 159:518-531. https://doi.org/10.1016/j. ahj.2009.12.027

10. Chatelain P, Arceo A, Rombaut E, Verin V, Urban P (1997) New device for compression of the radial artery after diagnostic and interventional cardiac procedures. Cathet Cardiovasc Diagn 40:297-300

11. Choi EY, Ko YG, Kim JB, Rhee J, Park S, Choi D, Jang Y, Shim WH, Cho SY (2005) Hemostatic efficacy of hydrophilic wound dressing after transradial catheterization. J Invasive Cardiol 17:459-462

12. Christopoulos G, Papayannis AC, Alomar M, Christakopoulos GE, Kotsia A, Michael TT, Rangan BV, Roesle M, Shorrock D, Makke L, Maragkoudakis S, Mohammad A, Sarode K, Chambers CE, Banerjee S, Brilakis ES (2016) Determinants of operator and patient radiation exposure during cardiac catheterization: Insights from the RadiCure (RADlation reduction during cardiac catheterization using real-timE monitoring) trial. Catheter Cardiovasc Interv 88:1046-1055. https:// doi.org/10.1002/ccd.26341

13. Cooper CJ, El-Shiekh RA, Cohen DJ, Blaesing L, Burket MW, Basu A, Moore JA (1999) Effect of transradial access on quality of life and cost of cardiac catheterization: A randomized comparison. Am Heart J 138:430-436

14. Dehghani P, Mohammad A, Bajaj R, Hong T, Suen CM, Sharieff W, Chisholm RJ, Kutryk MJ, Fam NP, Cheema AN (2009) Mechanism and predictors of failed transradial approach for percutaneous coronary interventions. Jacc Cardiovasc Interv 2:1057-1064. https://doi.org/10.1016/j.jcin.2009. 07.014

15. Dominici $M$, Diletti $R$, Milici $C$, Bock $C$, Placanica A, D'Alessandro G, Arrivi A, Italiani M, Buono E, Boschetti E (2013) Operator exposure to x-ray in left and right radial access during percutaneous coronary procedures: OPERA randomised study. Heart 99:480-484. https://doi.org/10.1136/ heartjnl-2012-302895

16. Greenwood MJ, Della-Siega AJ, Fretz EB, Kinloch $D$, Klinke P, Mildenberger R, Williams MB, Hilton $D$ (2005) Vascular communications of the hand in patients being considered for transradial coronary angiography: is the Allen's test accurate? J Am Coll Cardiol 46:2013-2017. https://doi.org/10.1016/j. jacc.2005.07.058

17. Hamon M, Pristipino C, Di Mario C, Nolan J, Ludwig J, Tubaro M, Sabate M, Mauri-Ferre J, Huber K, Niemela K, Haude M, Wijns W, Dudek D, Fajadet J, Kiemeneij F (2013) Consensus document on the radial approach in percutaneous cardiovascular interventions. Eurolntervention 8:1242-1251. https://doi.org/10.4244/eijv8i11a192 (Position paper by the European Association of Percutaneous Cardiovascular Interventions and Working Groups on Acute Cardiac Care** and Thrombosis of the European Society of Cardiology)

18. Ibanez B, James S, Agewall S, Antunes MJ, Bucciarelli-Ducci $C$, Bueno $H$, Caforio ALP, Crea F, Goudevenos JA, Halvorsen S, Hindricks G, Kastrati $A$, Lenzen $M J$, Prescott $E$, Roffi $M$, Valgimigli $M$,
Varenhorst C, Vranckx P, Widimsky P (2017) 2017 ESC Guidelines for the management of acute myocardial infarction in patients presenting with ST-segment elevation. Eur Heart J 39:119-177. https://doi.org/10.1093/eurheartj/ehx393

19. Jolly SS, Amlani S, Hamon M, Yusuf S, Mehta SR (2009) Radial versus femoral access for coronary angiography or intervention and the impact on major bleeding and ischemic events: a systematic review and meta-analysis of randomized trials. Am Heart J 157:132-140. https://doi.org/10.1016/j. ahj.2008.08.023

20. Kim SM, Kim DK, Kim DI, Kim DS, Joo SJ, Lee JW (2006) Novel diagnostic catheter specifically designed for both coronary arteries via the right transradial approach. A prospective, randomized trial of Tiger Il vs. Judkins catheters. Int J Cardiovasc Imaging 22:295-303. https://doi.org/10.1007/ s10554-005-9029-8

21. Lange HW, von Boetticher H (2006) Randomized comparison of operator radiation exposure during coronary angiography and intervention by radial or femoral approach. Catheter Cardiovasc Interv 67:12-16. https://doi.org/10.1002/ccd.20451

22. Langer $C$, Riehle J, Wuttig $H$, Durrwald $S$, Lange H, Samol A, Frey N, Wiemer M (2018) Efficacy of a one-catheter concept for transradial coronary angiography. PLoS ONE 13(30147):e189899. https://doi.org/10.1371/journal.pone.0189899

23. Lee JH, Kim MJ, Cha KS, Choi JH, Lee SY, Nam YH, Park JS, Chung SH, Kum DS, Park TH, Kim MH, Kim YD (2009) The feasibility of bypass graft angiography by right radial access. Korean Circ J 39:304-309. https://doi.org/10.4070/kcj.2009.39. 8.304

24. Louvard $Y$, Benamer $H$, Garot $P$, Hildick-Smith D, Loubeyre C, Rigattieri S, Monchi M, Lefevre T, Hamon M (2004) Comparison of transradial and transfemoral approaches for coronary angiography and angioplasty in octogenarians (the OCTOPLUS study). Am J Cardiol 94:1177-1180. https://doi.org/10.1016/j.amjcard.2004.07.089

25. Louvard Y, Lefevre T, Allain A, Morice M (2001) Coronary angiography through the radial or the femoral approach: The CARAFE study. Catheter Cardiovasc Interv 52:181-187

26. Lund C, Nes RB, Ugelstad TP, Due-Tonnessen $\mathrm{P}$, Andersen R, Hol PK, Brucher R, Russell D (2005) Cerebral emboli during left heart catheterization may cause acute brain injury. Eur Heart J 26:1269-1275. https://doi.org/10.1093/ eurheartj/ehi148

27. Mann JT 3rd, Cubeddu MG, Schneider JE, Arrowood M(1996) Right radial access for PTCA: a prospective study demonstrates reduced complications and hospital charges. J Invasive Cardiol 8(Suppl D):40D-44D

28. Mueller RL, Sanborn TA (1995) The history of interventional cardiology: cardiac catheterization, angioplasty, and related interventions. Am Heart J 129:146-172

29. Pancholy S, Coppola J, Patel T, Roke-Thomas M (2008) Prevention of radial artery occlusionpatent hemostasis evaluation trial (PROPHET study): a randomized comparison of traditional versus patency documented hemostasis after transradial catheterization. Catheter Cardiovasc Interv 72:335-340. https://doi.org/10.1002/ccd. 21639

30. Pancholy SB (2009) Comparison of the effect of intra-arterial versus intravenous heparin on radial artery occlusion after transradial catheterization. Am J Cardiol 104:1083-1085. https://doi.org/10. 1016/j.amjcard.2009.05.057 
31. Pancholy SB, Bernat I, Bertrand OF, Patel TM (2016) Prevention of radial artery occlusion after transradial catheterization: the PROPHET-II randomized trial. Jacc Cardiovasc Interv 9:1992-1999. https:// doi.org/10.1016/j.jcin.2016.07.020

32. Pancholy SB, Joshi P, Shah S, Rao SV, Bertrand OF, Patel TM (2015) Effect of vascular access site choice on radiation exposure during coronary Angiography: the REVERE trial (randomized evaluation of vascular entry site and radiation exposure). Jacc Cardiovasc Interv 8:1189-1196. https://doi.org/10.1016/j.jcin.2015.03.026

33. Pancholy SB, Patel G, Nanavaty SP, Pancholy MS (2017) Cardiogenic shock and access site choice. Minerva Cardioangiol 65(R05):74-80. https://doi. org/10.23736/S0026-4725.16.04246-8

34. Pancholy SB, Patel TM (2012) Effect of duration of hemostatic compression on radial artery occlusion after transradial access. Catheter Cardiovasc Interv 79:78-81.https://doi.org/10.1002/ccd.22963

35. Papadimos TJ, Hofmann JP (2002) Radial artery thrombosis, palmar arch systolic blood velocities, and chronic regional pain syndrome 1 following transradial cardiac catheterization. Catheter Cardiovasc Interv 57:537-540. https://doi.org/10. 1002/ccd.10367

36. Plourde G, Pancholy SB, Nolan J, Jolly S, Rao SV, Amhed I, Bangalore S, Patel T, Dahm JB, Bertrand OF (2015) Radiation exposure in relation to the arterial access site used for diagnostic coronary angiography and percutaneous coronary intervention: a systematic review and metaanalysis. Lancet 386:2192-2203. https://doi.org/ 10.1016/S0140-6736(15)00305-0

37. Rashid M, Kwok CS, Pancholy S, Chugh S, Kedev SA, Bernat I, Ratib K, Large A, Fraser D, Nolan J, Mamas MA (2016) Radial artery occlusion after Transradial interventions: a systematic review and Meta-analysis. J Am Heart Assoc 5. https://doi.org/ 10.1161/JAHA.115.002686

38. Rathore $S$, Stables RH, Pauriah M, Hakeem A Mills JD, Palmer ND, Perry RA, Morris JL (2010) A randomized comparison of TR band and radistop hemostatic compression devices after transradial coronary intervention. Catheter Cardiovasc Interv 76:660-667.https://doi.org/10.1002/ccd.22615

39. Roffi M, Patrono C, Collet JP, Mueller C, Valgimigli $M$, Andreotti F, Bax JJ, Borger MA, Brotons C, Chew DP, Gencer B, Hasenfuss G, Kjeldsen K, Lancellotti P, Landmesser U, Mehilli J, Mukherjee D, Storey RF, Windecker S, Baumgartner H, Gaemperli O, Achenbach S, Agewall S, Badimon L, Baigent $C$ Bueno H, Bugiardini R, Carerj S, Casselman F, Cuisset T, Erol C, Fitzsimons D, Halle M, Hamm C, Hildick-Smith D, Huber K, lliodromitis E, James $S$, Lewis BS, Lip GY, Piepoli MF, Richter D, Rosemann T, Sechtem U, Steg PG, Vrints C, Zamorano LJ (2016) 2015 ESC Guidelines for the management of acute coronary syndromes in patients presenting without persistent ST-segment elevation. Eur Heart J 37:267-315. https://doi.org/10.1093/ eurheartj/ehv320

40. Romagnoli E, Biondi-Zoccai G, Sciahbasi A, Politi L, Rigattieri S, Pendenza G, Summaria F, Patrizi R, Borghi A, Di Russo C, Moretti C, Agostoni P, Loschiavo P, Lioy E, Sheiban I, Sangiorgi G (2012) Radial versus femoral randomized investigation in ST-segment elevation acute coronary syndrome: the RIFLE-STEACS (Radial Versus Femoral Randomized Investigation in STElevation Acute Coronary Syndrome) study. J Am Coll Cardiol 60:2481-2489. https://doi.org/10. 1016/j.jacc.2012.06.017
41. Rosencher J, Chaib A, Barbou F, Arnould MA, Huber A, Salengro E, Jegou A, Allouch P, Zuily S, Mihoub F, Varenne O (2014) How to limit radial artery spasm during percutaneous coronary interventions: The spasmolytic agents to avoid spasm during transradial percutaneous coronary interventions (SPASM3) study. Catheter Cardiovasc Interv 84:766-771. https://doi.org/10.1002/ccd. 25163

42. Schiano P, Barbou F, Chenilleau MC, Louembe J, Monsegu J (2010) Adjusted weight anticoagulation for radial approach in elective coronarography: the AWARE coronarography study. Eurolntervention 6:247-250. https://doi.org/10. 4244/eijv6i2a39

43. Sciahbasi A, Rigattieri S, Sarandrea A, Cera M, Di Russo C, Fedele S, Romano S, Pugliese FR, Penco M (2015) Operator radiation exposure during right or left transradial coronary angiography: A phantom study. Cardiovasc Revasc Med 16:386-390. https:// doi.org/10.1016/j.carrev.2015.07.004

44. Sciahbasi A, Romagnoli E, Trani C, Burzotta F, Sarandrea A, Summaria F, Patrizi R, Rao S, Lioy E (2011) Operator radiation exposure during percutaneous coronary procedures through the left or right radial approach: the TALENT dosimetric substudy. Circ Cardiovasc Interv 4:226-231. https://doi.org/10.1161/circinterventions.111. 961185

45. Spaulding $C$, Lefevre T, Funck $F$, Thebault $B$ Chauveau M, Ben Hamda K, Chalet Y, Monsegu H, Tsocanakis O, Py A, Guillard N, Weber S (1996) Left radial approach for coronary angiography: results of a prospective study. Cathet Cardiovasc Diagn 39:365-370. https://doi.org/10.1002/(SICl)10970304(199612)39:4/365::AID-CCD8〉3.0.CO;2-B

46. Stella PR, Kiemeneij F, Laarman GJ, Odekerken D, Slagboom T, van der Wieken R (1997) Incidence and outcome of radial artery occlusion following transradial artery coronary angioplasty. Cathet Cardiovasc Diagn 40:156-158

47. Uhlemann M, Mobius-Winkler S, Mende M, Eitel I, Fuernau G, Sandri M, Adams V, Thiele H, Linke A Schuler G, Gielen S (2012) The Leipzig prospective vascular ultrasound registry in radial artery catheterization: impact of sheath size on vascula complications. Jacc Cardiovasc Interv 5:36-43. https://doi.org/10.1016/j.jcin.2011.08.011

48. Valgimigli M, Campo G, Penzo C, Tebaldi M, Biscaglia S, Ferrari R (2014) Transradial coronary catheterization and intervention across the whole spectrum of Allen test results. J Am Coll Cardiol 63:1833-1841. https://doi.org/10.1016/j.jacc. 2013.12.043

49. Valgimigli $M$, Gagnor A, Calabro P, Frigoli $E_{\text {, }}$ Leonardi S, Zaro T, Rubartelli P, Briguori C, Ando G, Repetto A, Limbruno U, Cortese B, Sganzerla P, Lupi A, Galli M, Colangelo S, lerna S, Ausiello A, Presbitero P, Sardella G, Varbella F, Esposito G, Santarelli A, Tresoldi S, Nazzaro M, Zingarelli A, de Cesare N, Rigattieri S, Tosi P, Palmieri C, Brugaletta S, Rao SV, Heg D, Rothenbuhler M, Vranckx P, Juni $P$, Investigators M (2015) Radial versus femoral access in patients with acute coronary syndromes undergoing invasive management: a randomised multicentre trial. Lancet 385:2465-2476. https:// doi.org/10.1016/S0140-6736(15)60292-6

50. Verheugt FW, Steinhubl SR, Hamon M, Darius $H$, Steg PG, Valgimigli M, Marso SP, Rao SV, Gershlick AH, Lincoff AM, Mehran R, Stone GW (2011) Incidence, prognostic impact, and influence of antithrombotic therapy on access and nonaccess site bleeding in percutaneous coronary intervention. Jacc Cardiovasc Interv 4:191-197. https://doi.org/10.1016/j.jcin.2010.10.011

51. Youssef AA, Hsieh YK, Cheng Cl, Wu CJ (2008) A single transradial guiding catheter for right and left coronary angiography and intervention. Eurolntervention 3:475-481

52. Zankl AR, Andrassy M, Volz C, Ivandic B, Krumsdorf U, Katus HA, Blessing E (2010) Radial artery thrombosis following transradial coronary angiography: incidence and rationale for treatment of symptomatic patients with low-molecular-weight heparins. Clin Res Cardiol 99:841-847. https://doi. org/10.1007/s00392-010-0197-8

53. Ziakas AG, Koskinas KC, Gavrilidis S, Giannoglou GD, Hadjimiltiades S, Gourassas I, Theofilogiannakos E, Economou F, Styliadis I (2010) Radial versus femoral access for orally anticoagulated patients. Catheter Cardiovasc Interv 76:493-499. https:// doi.org/10.1002/ccd.22527 